\title{
New Fixed Point Theorems of Mixed Monotone Operators
}

\author{
Xinsheng Du \\ School of Mathematics Sciences, Qufu Normal University, Qufu, China \\ Email: duxinsheng@mail.qfnu.edu.cn
}

Received August 24, 2013; revised September 24, 2013; accepted October 2, 2013

Copyright (C) 2014 Xinsheng Du. This is an open access article distributed under the Creative Commons Attribution License, which permits unrestricted use, distribution, and reproduction in any medium, provided the original work is properly cited. In accordance of the Creative Commons Attribution License all Copyrights (C) 2014 are reserved for SCIRP and the owner of the intellectual property Xinsheng Du. All Copyright (C) 2014 are guarded by law and by SCIRP as a guardian.

\begin{abstract}
Mixed monotone operator is an important nonlinear operator. It exists extensively in the research of nonlinear differential and integral equations. Generally, the research of mixed monotone operators in partially ordered Banach spaces requires compactness, continuity or concavity-convexity of the operators. In this paper, without any compact and continuous assumption, we obtain some new existence and uniqueness theorems of positive fixed point of e-concave-convex mixed monotone operators in Banach spaces partially ordered by a cone, which extends the existing corresponding results.
\end{abstract}

\section{KEYWORDS}

Mixed Monotone Operators; Cone; Partial Order

\section{Introduction}

Mixed monotone operators were introduced by Dajun Guo and V. Lakshmikantham in [1] in 1987. Thereafter, many authors have investigated these kinds of operators in Banach spaces and obtained a lot of interesting and important results. They are used extensively in nonlinear differential and integral equations. In this paper, without any compact and continuous assumption, we obtained some new existence and uniqueness theorems of positive fixed point of e-concave-convex mixed monotone operators in Banach spaces partially ordered by a cone.

Let the real Banach space $E$ be partially ordered by a cone $P$ of $E$, i.e., $x \leq y$ iff $y-x \in P$. $A: P \times P \rightarrow P$ is said to be a mixed monotone operator if $A(x, y)$ is increasing in $x$ and decreasing in $y$, i.e., $u_{i}, v_{i}(i=1,2) \in P, u_{1} \leq u_{2}, v_{1} \geq v_{2}$ implies. $A\left(u_{1}, v_{1}\right) \leq A\left(u_{2}, v_{2}\right)$. Element $x \in P$ is called a fixed point of $A$ iff $A(x, x)=x$.

Recall that cone $P$ is said to be solid if the interior $\stackrel{\circ}{P}$ is nonempty and we denote $x \gg \theta$ if $x \in \stackrel{\circ}{P}$. $P$ is normal if there exists a positive constant $N$, such that $\theta \leq x \leq y$ implies $\|x\| \leq N\|y\|, N$ is called the normal constant of $P$.

For all $x, y \in E$, the notation $x \sim y$ means that there exist $\lambda>0$ and $\mu>0$, such that $\lambda x \leq y \leq \mu x$. Clearly, $\sim$ is an equivalence relation. Given $e>\theta$, we denote by $P_{e}$ the set $P_{e}=\{x \in E \mid x \sim e\}$. It is easy to see that $P_{e} \subset P$ is convex and $\lambda P_{e}=P_{e}$ for all $\lambda>0$. If $P \neq \varnothing$ and $e \in P$, it is clear that $P_{e}=P$.

All the concepts discussed above can be found in [2-4]. For more facts about mixed monotone operators and other related concepts, the reader could refer to [5-7] and some of the reference therein.

\section{Main Results}

In this section, we present our main results. To begin with, we give the definition of e-concave-convex operators.

Definition 2.1. We say an operator $A: P_{e} \times P_{e} \rightarrow P_{e}$ is an e-concave-convex operators if there exist one positive function $\eta(t, u, v)$ such that 


$$
A\left(t u, \frac{1}{t} v\right) \geq t(1+\eta(t, u, v)) A(u, v), \forall u, v \in P_{e}, 0<t<1 .
$$

Remark 2.1. (2.1) implies that

$$
A\left(s u, \frac{1}{s} v\right) \leq s\left(1+\eta\left(\frac{1}{s}, s u, \frac{1}{s} v\right)\right)^{-1} A(u, v), \forall u, v \in P_{e}, s>1 .
$$

Theorem 2.1. Let $P$ be a normal cone of $E$, and let $A: P_{e} \times P_{e} \rightarrow P_{e}$ be a mixed monotone and e-concave-convex operator. In addition, suppose that there exist $u_{0}, v_{0} \in P_{e}, u_{0} \leq v_{0}$ (Since $u_{0}, v_{0} \in P_{e}$, we can choose a sufficiently small $\varepsilon \in(0,1)$ such that $\left.u_{0} \geq \varepsilon v_{0}\right)$ such that

$$
\begin{aligned}
& \left(H_{1}\right) u_{0} \leq A\left(u_{0}, v_{0}\right), A\left(v_{0}, u_{0}\right) \leq v_{0} \\
& \left(H_{2}\right) \forall t \in(0,1), \varlimsup_{n \rightarrow+\infty} n \eta\left(t, v_{n}, u_{n}\right) \geq \frac{1}{\varepsilon}-1,
\end{aligned}
$$

hold, where $u_{n}=A\left(u_{n-1}, v_{n-1}\right), v_{n}=A\left(v_{n-1}, u_{n-1}\right), n=1,2, \cdots$. Then $A$ has exactly one fixed pint $x^{*}$ in $P_{e}$. Moreover, constructing successively the sequence

$$
x_{n}=A\left(x_{n-1}, y_{n-1}\right), y_{n}=A\left(y_{n-1}, x_{n-1}\right), n=1,2, \cdots,
$$

for any initial $x_{0}, y_{0} \in P_{e}$, we have

$$
\left\|x_{n}-x^{*}\right\| \rightarrow 0,\left\|y_{n}-x^{*}\right\| \rightarrow 0, n \rightarrow \infty .
$$

Proof. We divide the proof into 3 steps.

Step 1. We prove $A$ has a fixed point in $P_{e}$.

Construct successively the sequences

$$
u_{n}=A\left(u_{n-1}, v_{n-1}\right), v_{n}=A\left(v_{n-1}, u_{n-1}\right), n=1,2, \cdots .
$$

It follows from $\left(H_{1}\right)$ and the mixed monotonicity of $A$ that

$$
u_{0} \leq u_{1} \leq \cdots \leq u_{n} \leq \cdots v_{n} \leq \cdots \leq v_{1} \leq v_{0} .
$$

Let

$$
r_{n}=\sup \left\{r>0 \mid u_{n} \geq r v_{n}\right\}, n=0,1,2, \cdots .
$$

Thus we have $u_{n} \geq r_{n} v_{n}, n=0,1,2, \cdots$, and then

$$
u_{n+1} \geq u_{n} \geq r_{n} v_{n} \geq r_{n} v_{n+1}, n=1,2, \cdots .
$$

Therefore, $r_{n+1} \geq r_{n}$, i.e., $\left\{r_{n}\right\}$ is increasing with $\left\{r_{n}\right\} \subset(0,1]$.

Suppose $r_{n} \rightarrow r^{*}$ as $n \rightarrow \infty$. Then $r^{*}=1$. Indeed, suppose to the contrary that $r_{n} \leq r^{*}<1$. By $\left(H_{1}\right)$, (2.1) and the mixed monotonicity of $A$, we have

$$
\begin{aligned}
u_{n+1} & =A\left(u_{n}, v_{n}\right) \geq A\left(r_{n} v_{n}, \frac{1}{r_{n}} u_{n}\right)=A\left(\frac{r_{n}}{r^{*}} r^{*} v_{n}, \frac{r^{*}}{r_{n}} \frac{1}{r^{*}} u_{n}\right) \geq \frac{r_{n}}{r^{*}} A\left(r^{*} v_{n}, \frac{1}{r^{*}} u_{n}\right) . \\
& \geq \frac{r_{n}}{r^{*}} r^{*}\left(1+\eta\left(r^{*}, v_{n}, u_{n}\right)\right) A\left(v_{n}, u_{n}\right)=r_{n}\left(1+\eta\left(r^{*}, v_{n}, u_{n}\right)\right) v_{n+1}
\end{aligned}
$$

Therefore

$$
\begin{aligned}
& r_{n+1} \geq r_{n}\left(1+\eta\left(r^{*}, v_{n}, u_{n}\right)\right), \\
& r_{n+1} \geq r_{0}\left(1+\eta\left(r^{*}, v_{0}, u_{0}\right)\right)\left(1+\eta\left(r^{*}, v_{1}, u_{1}\right)\right) \cdots\left(1+\eta\left(r^{*}, v_{n}, u_{n}\right)\right), n=0,1, \cdots
\end{aligned}
$$

By (2.1) and the mixed monotonicity of $A$, we know that $0<\eta(t, u, v) \leq \frac{1-t}{t}, \forall u, v \in P_{e}$, and thus $\varlimsup_{n \rightarrow+\infty} \eta\left(r^{*}, v_{n}, u_{n}\right)$ exists. We distinguish two cases. 
Case 1: $\varlimsup_{n \rightarrow+\infty} \eta\left(r^{*}, v_{n}, u_{n}\right)=\eta_{0} \neq 0$. In this case we know that $0<\eta_{0} \leq \frac{1-r^{*}}{r^{*}}$. So, we have $r^{*}=\varlimsup_{n \rightarrow+\infty} r_{n} \geq r^{*}\left(1+\eta_{0}\right)$, which is a contradiction.

Case 2: $\varlimsup_{n \rightarrow+\infty} \eta\left(r^{*}, v_{n}, u_{n}\right)=0$. In this case, it is easy to see that $\lim _{n \rightarrow+\infty} \eta\left(r^{*}, v_{n}, u_{n}\right)=0$. For convenience's sake, let $\eta_{n}=\eta\left(r^{*}, v_{n}, u_{n}\right)$. Since $\lim _{n \rightarrow+\infty} \eta_{n}=0$, there is a nonincreasing subsequence $\left\{\eta_{n k}\right\}$ of $\left\{\eta_{n}\right\}$ such that $\lim _{n \rightarrow+\infty} \eta_{n k}=0$. Without loss of generality, we may still use $\left\{\eta_{n}\right\}$ to stand for $\left\{\eta_{n k}\right\}$. From (2.5), we obtain

$$
r_{n+1} \geq r_{0}\left(1+\eta_{n}\right)^{n} \geq r_{0}\left(1+n \eta_{n}\right), n=0,1, \cdots
$$

Hence

$$
1>r^{*} \geq r_{0}\left(1+\varlimsup_{n \rightarrow+\infty} n \eta_{n}\right) \geq 1
$$

which is also a contradiction. Thus $r^{*}=1$.

For any natural number $p$ we have

$$
\begin{aligned}
& \theta \leq u_{n+p}-u_{n} \leq v_{n}-u_{n} \leq\left(1-r_{n}\right) v_{0}, \\
& \theta \leq v_{n+p}-v_{n} \leq v_{n}-u_{n} \leq\left(1-r_{n}\right) v_{0} .
\end{aligned}
$$

Since $P$ is normal, we have $\left\|u_{n+p}-u_{n}\right\| \leq N\left(1-r_{n}\right)\left\|v_{0}\right\|,\left\|v_{n+p}-v_{n}\right\| \leq N\left(1-r_{n}\right)\left\|v_{0}\right\|$. Here $N$ is the normality constant.

So $\left\{u_{n}\right\}$ and $\left\{v_{n}\right\}$ are Cauchy sequences. Because $E$ is complete, there exist $u^{*}, v^{*}$ such that $u_{n} \rightarrow u^{*}, v_{n} \rightarrow v^{*}(n \rightarrow+\infty)$, By (2.4) we know that $u_{n} \leq u^{*} \leq v^{*} \leq v_{n}$ and

$$
\theta \leq v^{*}-u^{*} \leq\left(1-r_{n}\right) v_{0} \text {. }
$$

Further

$$
\left\|v^{*}-u^{*}\right\| \leq N\left(1-r_{n}\right)\left\|v_{0}\right\|,
$$

And thus $v^{*}=u^{*}$. Let $x^{*}:=v^{*}=u^{*}$, we obtain

$$
u_{n+1}=A\left(u_{n}, v_{n}\right) \leq A\left(x^{*}, x^{*}\right) \leq A\left(v_{n}, u_{n}\right)=v_{n+1} .
$$

Let $n \rightarrow+\infty$, we get $x^{*}=A\left(x^{*}, x^{*}\right)$. That is, $x^{*}$ is a fixed point of $A$ in $P_{e}$.

Step 2. We prove that $x^{*}$ is the unique fixed point of $A$ in $P_{e}$.

In fact, suppose $\bar{x}$ is another fixed point of $A$ in $P_{e}$. Since $\bar{x}, x^{*} \in P_{e}$, there exist positive numbers $\alpha, \beta$ such that $x^{*} \geq \alpha \bar{x}, \bar{x} \geq \beta x^{*}$. Let

$$
\gamma_{0}=\sup \left\{\gamma>0 \mid \gamma \bar{x} \leq x^{*} \leq \frac{1}{\gamma} \bar{x}\right\} .
$$

Evidently, $\gamma_{0} \in(0,1]$. We now prove $\gamma_{0}=1$. If otherwise, $0<\gamma_{0}<1$. From (2.1), we obtain

$$
\begin{aligned}
& x^{*}=A\left(x^{*}, x^{*}\right) \geq A\left(\gamma_{0} \bar{x}, \frac{1}{\gamma_{0}} \bar{x}\right) \geq \gamma_{0}\left(1+\eta\left(\gamma_{0}, \bar{x}, \bar{x}\right)\right) A(\bar{x}, \bar{x})=\gamma_{0}\left(1+\eta\left(\gamma_{0}, \bar{x}, \bar{x}\right)\right) \bar{x} \\
& \bar{x}=A(\bar{x}, \bar{x}) \geq A\left(\gamma_{0} x^{*}, \frac{1}{\gamma_{0}} x^{*}\right) \geq \gamma_{0}\left(1+\eta\left(\gamma_{0}, x^{*}, x^{*}\right)\right) A\left(x^{*}, x^{*}\right)=\gamma_{0}\left(1+\eta\left(\gamma_{0}, x^{*}, x^{*}\right)\right) x^{*} .
\end{aligned}
$$

Which contradicts the definition of $\gamma_{0}$. Hence $\gamma_{0}=1$, thus $x^{*}=\bar{x}$. Therefore $A$ has a unique fixed point $x^{*}$ in $P_{e}$.

Step 3. We prove (2.3).

For any $x_{0}, y_{0} \in P_{e}$, we can choose a small number $t_{0} \in(0,1)$ such that

$$
t_{0} x^{*} \leq x_{0} \leq \frac{1}{t_{0}} x^{*}, t_{0} x^{*} \leq y_{0} \leq \frac{1}{t_{0}} x^{*} .
$$


Let

$$
a_{0}=t_{0} x^{*}, b_{0}=\frac{1}{t_{0}} x^{*}, a_{n}=A\left(a_{n-1}, b_{n-1}\right), b_{n}=A\left(b_{n-1}, a_{n-1}\right) .
$$

Using (2.7), (2.8) and the mixed monotonicity of $A$, we have

$$
\begin{aligned}
& a_{n} \leq x^{*} \leq b_{n}, a_{n} \leq x_{n} \leq b_{n}, a_{n} \leq y_{n} \leq b_{n}, n=1,2, \cdots \\
& a_{0} \leq a_{1} \leq \cdots \leq a_{n} \leq \cdots \leq b_{n} \leq \cdots \leq b_{1} \leq b_{0} .
\end{aligned}
$$

Let

$$
\lambda_{n}=\sup \left\{t>0 \mid t x^{*} \leq a_{n}, t b_{n} \leq x^{*}\right\}, n=1,2, \cdots .
$$

From (2.8) and (2.9), we obtain

$$
\begin{aligned}
& \lambda_{n} x^{*} \leq a_{n}, \lambda_{n} b_{n} \leq x^{*}, n=1,2, \cdots, \\
& 0 \leq t_{0}=\lambda_{0} \leq \lambda_{1} \leq \cdots \leq \lambda_{n} \leq \cdots \leq 1 .
\end{aligned}
$$

In what follows, we will prove that $\lim _{n \rightarrow \infty} \lambda_{n}=1$. If not, that is, $\lim _{n \rightarrow \infty} \lambda_{n}=\lambda^{*}<1$, then from (2.8) and (2.11), we have

$$
\begin{aligned}
a_{n+1} & =A\left(a_{n}, b_{n}\right) \geq A\left(\lambda_{n} x^{*}, \frac{1}{\lambda_{n}} x^{*}\right)=A\left(\frac{\lambda_{n}}{\lambda^{*}} \lambda^{*} x^{*}, \frac{\lambda^{*}}{\lambda_{n}} \frac{1}{\lambda^{*}} x^{*}\right) \\
& \geq \frac{\lambda_{n}}{\lambda^{*}} A\left(\lambda^{*} x^{*}, \frac{1}{\lambda^{*}} x^{*}\right) \geq \lambda_{n}\left(1+\eta\left(\lambda^{*}, x^{*}, x^{*}\right)\right) x^{*} ; \\
b_{n+1}= & A\left(b_{n}, a_{n}\right) \leq A\left(\frac{1}{\lambda_{n}} x^{*}, \lambda_{n} x^{*}\right)=A\left(\frac{\lambda^{*}}{\lambda_{n}} \frac{1}{\lambda^{*}} x^{*}, \frac{\lambda_{n}}{\lambda^{*}} \lambda^{*} x^{*}\right) \\
& \leq \frac{\lambda^{*}}{\lambda_{n}} A\left(\frac{1}{\lambda^{*}} x^{*}, \lambda^{*} x^{*}\right) \leq \frac{1}{\lambda_{n}}\left(1+\eta\left(\lambda^{*}, \frac{1}{\lambda^{*}} x^{*}, \lambda^{*} x^{*}\right)\right)^{-1} x^{*}
\end{aligned}
$$

Let $d=\min \left\{\eta\left(\lambda^{*}, x^{*}, x^{*}\right), \eta\left(\lambda^{*}, \frac{1}{\lambda^{*}} x^{*}, \lambda^{*} x^{*}\right)\right\}$. It follows from (2.11), (2.12) and (2.13) that $\lambda_{n+1} \geq \lambda_{n}(1+d) \geq \cdots \geq \lambda_{1}(1+d)^{n}$, which is a contradiction. That is $\lim _{n \rightarrow \infty} \lambda_{n}=1$.

From (2.9) and (2.11), we have

$$
\lambda_{n} x^{*} \leq x_{n} \leq \frac{1}{\lambda_{n}} x^{*}, \lambda_{n} x^{*} \leq y_{n} \leq \frac{1}{\lambda_{n}} x^{*}, n=1,2, \cdots
$$

Thus,

$$
\left(\lambda_{n}-1\right) x^{*} \leq x_{n}-x^{*} \leq\left(\frac{1}{\lambda_{n}}-1\right) x^{*},\left(\lambda_{n}-1\right) x^{*} \leq y_{n}-x^{*} \leq\left(\frac{1}{\lambda_{n}}-1\right) x^{*}, n=1,2, \cdots .
$$

By using the normality of $P$, we know that (2.2) holds.

\section{Concerned Remarks and Corollaries}

Using Theorem 2.1, we have the following corollaries.

Corollary 3.1. Let $P$ be a normal cone of $E$, and let $A: P_{e} \times P_{e} \rightarrow P_{e}$ be a mixed monotone and e-concaveconvex operator. In addition, suppose that there exist $u_{0}, v_{0} \in P_{e}, u_{0} \leq v_{0}$ (Since $u_{0}, v_{0} \in P_{e}$, we can choose a sufficiently small $\varepsilon \in(0,1)$, such that $\left.u_{0} \geq \varepsilon v_{0}\right)$, such that

$$
\begin{aligned}
& \left(H_{1}\right) u_{0} \leq A\left(u_{0}, v_{0}\right), A\left(v_{0}, u_{0}\right) \leq v_{0} ; \\
& \left(H_{2}^{\prime}\right) \forall t \in(0,1), \varlimsup_{n \rightarrow+\infty} n \eta\left(t, v_{n}, u_{n}\right)=+\infty,
\end{aligned}
$$


hold, where $u_{n}=A\left(u_{n-1}, v_{n-1}\right), v_{n}=A\left(v_{n-1}, u_{n-1}\right), n=1,2, \cdots$. Then $A$ has exactly one fixed pint $x^{*}$ in $P_{e}$. Moreover, constructing successively the sequence

$$
x_{n}=A\left(x_{n-1}, y_{n-1}\right), y_{n}=A\left(y_{n-1}, x_{n-1}\right), n=1,2, \cdots,
$$

for any initial $x_{0}, y_{0} \in P_{e}$, we have

$$
\left\|x_{n}-x^{*}\right\| \rightarrow 0,\left\|y_{n}-x^{*}\right\| \rightarrow 0, n \rightarrow \infty .
$$

Corollary 3.2. Let $P$ be a normal cone of $E$, and let $A: P_{e} \times P_{e} \rightarrow P_{e}$ be a mixed monotone and e-concaveconvex operator. In addition, suppose that

$\left(H_{3}\right)$ there exist $u_{0}, v_{0} \in P_{e}$, such that $u_{0} \leq A\left(u_{0}, v_{0}\right), A\left(v_{0}, u_{0}\right) \leq v_{0}$;

$\left(H_{4}\right)$ for all $t \in(0,1)$, there exist $x_{t}, y_{t} \in\left[u_{0}, v_{0}\right]$, such that $\eta(t, x, y) \geq \eta\left(t, x_{t}, y_{t}\right), \quad \forall x, y \in P_{e}$ hold.

Then $A$ has exactly one fixed pint $x^{*}$ in $P_{e}$. Moreover, constructing successively the sequence

$$
x_{n}=A\left(x_{n-1}, y_{n-1}\right), y_{n}=A\left(y_{n-1}, x_{n-1}\right), n=1,2, \cdots,
$$

for any initial $x_{0}, y_{0} \in P_{e}$, we have

$$
\left\|x_{n}-x^{*}\right\| \rightarrow 0,\left\|y_{n}-x^{*}\right\| \rightarrow 0, n \rightarrow \infty .
$$

Corollary 3.3. Let $P$ be a normal cone of $E$, and let $A: P_{e} \times P_{e} \rightarrow P_{e}$ be a mixed monotone and e-concaveconvex operator. In addition, suppose that $\forall t \in(0,1), \eta(t, x, y)$ are monotone on $x$ and $y$. Then a necessary and sufficient condition for $A$ to have exactly one fixed pint $x^{*}$ in $P_{e}$ is that $\left(H_{3}\right)$ holds. Moreover, constructing successively the sequence

$$
x_{n}=A\left(x_{n-1}, y_{n-1}\right), y_{n}=A\left(y_{n-1}, x_{n-1}\right), n=1,2, \cdots,
$$

for any initial $x_{0}, y_{0} \in P_{e}$, we have

$$
\left\|x_{n}-x^{*}\right\| \rightarrow 0,\left\|y_{n}-x^{*}\right\| \rightarrow 0, n \rightarrow \infty .
$$

Proof. Corollary 3.1 ensures the sufficiency of Corollary 2.3, so we have only to prove the necessity of Corollary 2.3.

Suppose that $x^{*}$ is the unique fixed point of $A$ in $P_{e}$. For any $t_{0} \in(0,1)$, let $u_{0}=t_{0} x^{*}, v_{0}=\frac{1}{t_{0}} x^{*}$. It follows from (1.1), (1.2) and the mixed monotonicity of $A$ that

$$
\begin{aligned}
& A\left(u_{0}, v_{0}\right)=A\left(t_{0} x^{*}, \frac{1}{t_{0}} x^{*}\right) \geq t_{0}\left(1+\eta\left(t_{0}, x^{*}, x^{*}\right)\right) A\left(x^{*}, x^{*}\right) \geq t_{0} x^{*}=u_{0} \\
& A\left(v_{0}, u_{0}\right)=A\left(\frac{1}{t_{0}} x^{*}, t_{0} x^{*}\right) \leq \frac{1}{t_{0}}\left(1+\eta\left(t_{0}, \frac{1}{t_{0}} x^{*}, t_{0} x^{*}\right)\right)^{-1} A\left(x^{*}, x^{*}\right) \leq \frac{1}{t_{0}} x^{*}=v_{0} .
\end{aligned}
$$

Therefore, $\left(H_{3}\right)$ holds.

Corollary 3.4. Let $P$ be a normal cone of $E$, and let $A: P_{e} \times P_{e} \rightarrow P_{e}$ be a mixed monotone operator. In addition, suppose that $\forall t \in(0,1)$, there exists $\eta(t)>0$ such that

$$
A\left(t x, \frac{1}{t} y\right) \geq t(1+\eta(t)) A(x, y), x, y \in P_{e},
$$

holds. Then a necessary and sufficient condition for $A$ to have exactly one fixed pint $x^{*}$ in $P_{e}$ is that $\left(H_{3}\right)$ holds. Moreover, constructing successively the sequence

$$
x_{n}=A\left(x_{n-1}, y_{n-1}\right), y_{n}=A\left(y_{n-1}, x_{n-1}\right), n=1,2, \cdots,
$$

for any initial $x_{0}, y_{0} \in P_{e}$, we have

$$
\left\|x_{n}-x^{*}\right\| \rightarrow 0,\left\|y_{n}-x^{*}\right\| \rightarrow 0, n \rightarrow \infty .
$$

Corollary 3.5. Let $P$ be a normal cone of $E$, and let $A: P_{e} \times P_{e} \rightarrow P_{e}$ be a mixed monotone operator with 
property (2.1). In addition, suppose that $\lim _{t \rightarrow 0^{+}} \eta(t)=+\infty$ holds. Then $A$ has exactly one fixed pint $x^{*}$ in $P_{e}$.

Proof. In fact, by corollary 2.4 , we have only to prove that $\left(H_{3}\right)$ holds.

For any $x_{0} \in P_{e}$, it follows from $\lim _{t \rightarrow 0^{+}} \eta(t)=+\infty$ that there exists $t_{0} \in(0,1)$, such that $\frac{1}{1+\eta\left(t_{0}\right)} x_{0} \leq A\left(x_{0}, x_{0}\right) \leq\left(1+\eta\left(t_{0}\right)\right) x_{0}$. Let $u_{0}=t_{0} x_{0}, v_{0}=\frac{1}{t_{0}} x_{0}$. Following from (3.1) and the mixed monotonicity of $A$, we get

$$
\begin{aligned}
& A\left(u_{0}, v_{0}\right)=A\left(t_{0} x_{0}, \frac{1}{t_{0}} x_{0}\right) \geq t_{0}\left(1+\eta\left(t_{0}\right)\right) A\left(x_{0}, x_{0}\right) \geq t_{0} x_{0}=u_{0} ; \\
& A\left(v_{0}, u_{0}\right)=A\left(\frac{1}{t_{0}} x_{0}, t_{0} x_{0}\right) \leq \frac{1}{t_{0}}\left(1+\eta\left(t_{0}\right)\right)^{-1} A\left(x_{0}, x_{0}\right) \leq \frac{1}{t_{0}} x_{0}=v_{0} .
\end{aligned}
$$

The Corollary 3.5 is thus proved.

\section{Acknowledgements}

The author thanks the referee for his valuable comments and suggestions. This paper was supported by SRFDP (NO. 20103705120002).

\section{REFERENCES}

[1] D. J. Guo and V. Lakskmikantham, “Coupled Fixed Points of Mixed Monotone Operator with Applications,” Nonlinear Analysis, Vol. 11, No. 5, 1987, pp. 623-632.

[2] K. Deiming, “Nonlinear Functional Analysis,” Springer-Verlag, New York, 1985.

[3] D. J. Guo and V. Lakskmikantham, “Nonlinear Problems in Abstract Cones,” Acadamic Press, Boston, 1988.

[4] D. J. Guo, “Nonlinear Functional Analysis,” 5th Edition, Shandong Scientific Technical Publishers, Jinan, 2000.

[5] D. J. Guo, "Fixed Points of Mixed Monotone Operators with Applications," Journal of Mathematical Analysis and Applications, Vol. 31, 1988, pp. 215-224.

[6] Y. X. Wu and Z. D. Liang, "Existence and Uniqueness of Fixed Points for Mixed Monotone Operators,” Nonlinear Analysis, Vol. 65, 2006, pp. 1913-1924.

[7] Z. Q. Zhao, "Existence and Uniqueness of Fixed Points for Some Mixed Monotone Operators,” Nonlinear Analysis, Vol. 73, 2010, pp. 1481-1490. 